\title{
Producción de ácido giberélico a partir de Gibberella fujikuroi utilizando lodo residual municipal como sustrato
}

\author{
Irene Cuali-Álvarez, Sergio H. Pavón-Romero, Arturo Colín-Cruz* \\ Universidad Autónoma del Estado de México, Facultad de Química, Laboratorio de Química Ambiental. \\ Paseo Colón y Tollocan S/N Col. Residencial Colón, C.P. 50180. Toluca, Edo. de México. \\ *acolinc@uaemex.mx
}

Recibido: 23-11-2010; Aceptado: 04-03-2011

\begin{abstract}
Resumen
Objetivo. Utilizar lodos residuales municipales (LRM) provenientes de una planta de tratamiento de aguas residuales ubicada en Toluca, Estado de México, para cultivar al hongo Gibberella fujikuroi en fermentación sumergida y producir ácido giberélico $\left(\mathrm{AG}_{3}\right)$. Materiales y métodos. Se utilizó Gibberella fujikuroi (CDBB:268). Para la obtención de $\mathrm{AG}_{3}$ se verificó la producción empleando como sustrato al medio de cultivo estándar (MCE). La determinación de $\left(\mathrm{AG}_{3}\right)$ fue por cromatografía líquida de alta resolución (HPLC) con un equipo Varian, 9050,9012. Se obtuvieron 6 muestras de lodos de una planta tratadora de aguas residuales en Toluca, Estado de México y fueron caracterizados. Finalmente ambos sustratos (LRM y MCE) se usaron en fermentación sumergida y por extracción se obtuvo el AG $_{3}$ cuantificado por HPLC. Resultados. La caracterización del LRM demostró que el contenido de materia orgánica (MO) es de 5.20 \% (m/v) y nitrógeno total $\left(\mathrm{N}_{\mathrm{T}}\right)$ de $0.25 \%(\mathrm{~m} / \mathrm{v})$, dicha composición está dentro del rango como sustrato para la producción del $\mathrm{AG}_{3}$ por Gibberella fujikuroi. El hongo se cultivó durante 3, 8, 13 y 30 días utilizando lodo residual estéril con una humedad del 95.6\%(m/v) y en medio de cultivo estándar (MCE), las muestras se procesaron y analizaron por cromatografía líquida de alta resolución (HPLC), donde la producción de $\mathrm{AG}_{3}$ en el LRM fue de $460.06 \mathrm{mg} / \mathrm{L}$ para 30 días en fermentación sumergida a un pH de 4.0 y $1014.46 \mathrm{mg} / \mathrm{L}$ para el control. Conclusiones. El contenido nutritivo del LRM es adecuado para el crecimiento del hongo Gibberella fujikuroi y la producción de $\mathrm{AG}_{3}$ empleando como sustrato LRM.
\end{abstract}

Palabras clave: ácido giberélico, Gibberella fujikuroi, lodo residual

\begin{abstract}
Production of gibberellic acid from Gibberella fujikuroi using municipal sewage sludge as a substrate. Objective. To use municipal sewage sludge (LRM) from a wastewater treatment plant located in Toluca, State of Mexico, to grow the fungus Gibberella fujikuroi in submerged fermentation and to produce gibberellic acid $\left(\mathrm{AG}_{3}\right)$. Materials and methods. We used Gibberella fujikuroi (CDBB: 268). To obtain $\mathrm{AG}_{3}$, production was verified using as a substrate the standard culture medium (MCE). Gibberellic acid determination was done with high performance liquid chromatography (HPLC) with a Varian 9050.9012 equipment. We obtained 6 samples of sludge from a wastewater treatment plant in Toluca, State of Mexico, that were then characterized. Finally, both substrates (LRM and MCE) were used in submerged fermentation, and $\mathrm{GA}_{3}$ was obtained by extraction and quantified using HPLC. Results. The LRM characterization showed that the organic matter content $(\mathrm{MO})$ is of $5.20 \%(\mathrm{w} / \mathrm{v})$ and the total nitrogen content $\left(\mathrm{N}_{\mathrm{T}}\right)$ is of $0.25 \%(\mathrm{w} / \mathrm{v})$. Such composition is within the range as a substrate for the production of $\mathrm{AG}_{3}$ by Gibberella fujikuroi. The fungus was cultivated for $3,8,13$ and 30 days in sterile sewage sludge with a moisture content of $95.6 \%(\mathrm{w} / \mathrm{v})$ and in standard culture medium (MCE). Samples were processed and analyzed by high performance liquid chromatography (HPLC). Production of $\mathrm{AG}_{3}$ in the LRM was of $460.06 \mathrm{mg} / \mathrm{L}$ after 30 days in submerged fermentation at $\mathrm{pH} 4.0$, and of $1,014.46 \mathrm{mg} / \mathrm{L}$ in the control. Conclusion. The nutrient content of LRM is suitable for the growth of the fungus Gibberella fujikuroi and for the production of $\mathrm{GA}_{3}$ when used as a substrate.
\end{abstract}

Key words: gibberellic acid, Gibberella fujikuroi, sewage sludge 


\section{Resumo}

Produção de ácido giberélico desde Gibberella fujikuroi usando lodo residual municipal como substrato. Objetivo. Usar lodos residuais municipais (LRM) procedentes de uma estação de tratamento de águas residuais de Toluca, Estado de México, para cultivar o fungo Gibberella fujikuroi em fermentação submersa e produzir ácido giberélico $\left(\mathrm{AG}_{3}\right)$. Materiais e métodos. Foi utilizado Gibberella fujikuroi (CDBB: 268). Para obter $\mathrm{AG}_{3}$ foi verificada a produção empregando como substrato o meio de cultura padrão (MCE). A determinação de ácido giberélico foi por cromatografia líquida de alta eficiência (HPLC) com um equipo Varian, 9050,9012. Foram obtidas seis amostras de lodos de uma estação de tratamento de águas residuais em Toluca, Estado de México e foram caracterizadas. Finalmente,

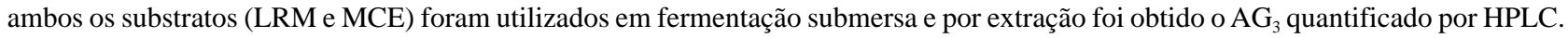
Resultados. A caracterização do LRM demonstrou que o teor de matéria orgânica (MO) é 5,20\% (m/v) e o nitrogênio total $\left(\mathrm{N}_{\mathrm{T}}\right) 0,25 \%(\mathrm{~m} / \mathrm{v})$, esta composição está dentro do intervalo como substrato para a produção de $\mathrm{AG}_{3}$ por Gibberella fujikuroi. O fungo foi cultivado por 3,8 , 13 e 30 dias usando lodo residual estéril com um teor de umidade de $95,6 \%$ (m/v) e em meio de cultura padrão (MEC), as amostras foram processadas e analisadas por cromatografia líquida de alta resolução (HPLC), onde a produção de AG $_{3}$ no LRM foi 460,06 mg/L para 30 dias em fermentação submersa em pH 4,0 e 1.014,46 mg/L para o controle. Conclusões. O teor nutritivo do LRM é adequado para o crescimento do fungo Gibberella fujikuroi e a produção de $\mathrm{AG}_{3}$, empregando como substrato o LRM.

Palavras-chave: ácido giberélico, Gibberella fujikuroi, lodo residual.

\section{Introducción}

De las 136 giberelinas que han sido identificadas en plantas, hongos y bacterias, es el ácido giberélico $\left(\mathrm{AG}_{3}\right)$ la giberelina más importante $(1,2)$. Entre las aplicaciones de esta fitohormona se encuentra la contribución sobre los procesos de malteado de la cebada, su actividad como promotor del desarrollo de los racimos de uva, su labor en los procesos de fecundación de ciertos frutos, el aumento que genera en las distancias internodales de algunas plantas, su actividad como desencadenante de la floración en otras, su oposición al efecto inhibidor de la oscuridad en el desarrollo de algunos tejidos vegetales, la reducción en los periodos de latencia de semillas (3).

El ácido giberélico se produce por microorganismos, siendo el hongo ascomiceto Gibberella fujikuroi el más empleado para su producción. Este hongo es una especie pleomórfica, ya que puede tener una o más formas en sus estados sexuales (perfecto) como en los asexuales (imperfectos). Al estado imperfecto del hongo se le llama Fusarium moniliforme y al estado perfecto Gibberella fujikuroi (4).

Los metabolitos producidos por Gibberella fujikuroi son numerosos, siendo el bikaverin y las giberelinas los más conocidos. Para la producción de las giberelinas, especialmente $\mathrm{GA}_{3}$ se han empleado diversos procesos. Pierotty et al. (3), mencionan que se han usado distintas técnicas de fermentación a nivel laboratorio, tales como los trabajos de fermentaciones sumergidas realizados por Borrow et al. (1964), investigaciones en células inmovilizadas por Jones y Pharis, (1987), ensayos con sistema de inmovilización basados en fibras poliméricas y diversos sistemas de fermentación en fase sólida realizados por Lu et al. (1995) y Gelmi et al. (2000 y 2002).
Uno de los campos de la Biotecnología que ha logrado una mayor evolución en el último siglo, es la producción de metabolitos secundarios, hoy en día se produce una gran cantidad de estas sustancias, las cuales son de alto valor agregado, y cuya mayoría provienen de hongos (3).

Para producir $\mathrm{AG}_{3}$ se han empleado distintos sustratos en el crecimiento del microorganismo y producción de la fitohormona. Gelmi et al. (5), empleó Amberlita IRA-900, una resina sintética a diferentes condiciones de temperatura y actividad del agua, utilizando urea como única fuente de nitrógeno. La cáscara de café y bagazo de cassava fueron empleados por M. Machado et al. (1) en la fermentación a diferentes tiempos, estos residuos agroindustriales proporcionaron las diferentes fuentes de carbono. Durán et al. (6) y Nhujak et al. (7) emplearon medios de cultivo sintéticos. Indicando que las concentraciones bajas de nitrógeno favorecen la producción del ácido. También se han empleado biocatalizadores soportados en matrices porosas para la producción de $\mathrm{AG}_{3}$, en un medio nutritivo preparado a partir de sus componentes para el crecimiento del micelio y producción del ácido giberélico (3). Como se hace referencia, algunos autores utilizan residuos sólidos como sustrato para el crecimiento de Gibberella fujikuroi y producir $\mathrm{AG}_{3}$, en este caso se utilizó un lodo residual proveniente de un sistema de tratamiento de aguas residuales, esto es importante, porque a partir de un residuo se obtiene un recurso (8).

Los lodos se clasifican en base a las aguas residuales que los originan, por lo tanto, se obtienen lodos residuales industriales, municipales y mixtos. En México no existe una cifra oficial reportada sobre la producción de lodos generados. Colín et al. (9) reportaron una producción de 12 millones de toneladas/año y son muy pocas las plantas que realizan algún proceso de tratamiento y disposición final, ya que generalmente, carecen de las instalaciones 
para llevar a cabo el manejo adecuado de los lodos generados (10). Para dar solución al problema, se han realizado estudios que proponen alternativas de uso; Rulkens (11) propone que los lodos residuales municipales pueden ser aprovechados para producir energía, Teuber et al. (12) menciona que el aprovechamiento de diferentes residuos orgánicos (desechos urbanos, lodos acuícolas, entre otros) ha aumentado exponencialmente, debido a su valor agronómico y a su capacidad para aumentar la calidad y cantidad de materia orgánica, por su valor como enmienda y su posibilidad de incrementar el contenido de nutrientes en el suelo (principalmente fósforo, calcio y magnesio). Dirkzwager et al. (13) analizaron lodos residuales municipales en España y encontraron una concentración de materia orgánica de $53.8 \%$, nitrógeno $3.18 \%$, potasio $0.14 \%$ y fósforo $3.96 \%$. Colín-Cruz (14), analizó lodos residuales municipales en México, obteniendo en relación a materia orgánica $62.5 \%$, de nitrógeno $2.1 \%$, de potasio $1.2 \%$ y de fósforo $0.1 \%$, ambos autores concluyeron que estos lodos son útiles como mejoradores del suelo y como fuente de nutrientes para vegetales.

La concentración de nutrientes que presentan los lodos residuales puede ser aprovechada como medio de cultivo para ciertos microorganismos, como por ejemplo para la producción de Bacillus thuringiensis variedad kurstaki (19), ya que el contenido de materia orgánica y nitrógeno pueden ser utilizados como sustrato, otro ejemplo es el trabajo realizado por Ayesteran (8), en el cual, utilizó lodos residuales industriales para el crecimiento del hongo Gibberella fujikuroi y la producción de ácido giberélico, obteniendo $38 \mathrm{mg} / \mathrm{L}$ del ácido en 4 días de cultivo, demostrando que estos residuos pueden emplearse como sustrato para dicho microorganismo. Por lo anterior, el propósito de este trabajo fue utilizar lodos residuales municipales para la producción de ácido giberélico empleando al hongo Gibberella fujikuroi mediante el proceso de fermentación sumergida.

\section{Materiales y métodos}

\section{Cepa}

El microorganismo empleado para la producción de $\mathrm{AG}_{3}$ fue la cepa de Gibberella fujikuroi (CDBB:268), conservada a $4^{\circ} \mathrm{C}$ y resembrada cada 20 días en tubos inclinados con Agar Papa Dextrosa (PDA) a $28^{\circ} \mathrm{C}$. Se identificaron las características macroscópicas del hongo observando las colonias presentes en los tubos de cultivo y las características microscópicas se determinaron por medio de microcultivos.

\section{Producción de ácido giberélico en fermentación sumergida}

Se verificó que la cepa identificada como Gibberella fujikuroi produce $\mathrm{AG}_{3}$ empleando como sustrato el MCE, el cual, se preparó en un matraz Erlenmeyer de $250 \mathrm{~mL}$ conteniendo $100 \mathrm{~mL}$ compuesto por: Glucosa $(80 \mathrm{~g} / \mathrm{L})$, $\mathrm{MgSO}_{4} .7 \mathrm{H}_{2} \mathrm{O}(0.45 \mathrm{~g} / \mathrm{L}), \mathrm{KH}_{2} \mathrm{PO}_{4}(5 \mathrm{~g} / \mathrm{L}), \mathrm{NH}_{4} \mathrm{NO}_{3}(1.85 \mathrm{~g} / \mathrm{L})$, $\mathrm{FeSO}_{4} .7 \mathrm{H}_{2} \mathrm{O}(0.2 \mathrm{~g} / \mathrm{L}), \mathrm{ZnSO}_{4} .7 \mathrm{H}_{2} \mathrm{O}(0.2 \mathrm{~g} / \mathrm{L}), \mathrm{CaCl}_{2} .2 \mathrm{H}_{2} \mathrm{O}$ $(0.1 \mathrm{~g} / \mathrm{L}), \mathrm{CuSO}_{4}(0.02 \mathrm{~g} / \mathrm{L}), \mathrm{CoCl}_{2}(0.02 \mathrm{~g} / \mathrm{L})$, $\mathrm{Na}_{2} \mathrm{~B}_{4} \mathrm{O}_{7} \cdot 10 \mathrm{H}_{2} \mathrm{O}(0.02 \mathrm{~g} / \mathrm{L}), \mathrm{Na}_{2} \mathrm{MoO}_{4} \cdot 2 \mathrm{H}_{2} \mathrm{O}(0.02 \mathrm{~g} / \mathrm{L})$, $\mathrm{MnSO}_{4} \cdot \mathrm{H}_{2} \mathrm{O}(0.02 \mathrm{~g} / \mathrm{L})$, etilendiaminotetraacético (0.6 g/ L), se esterilizó durante 15 minutos a $15 \mathrm{lb}$ y posteriormente se inoculó con el contenido de un tubo de cultivo en baño maría durante 7 días a $28^{\circ} \mathrm{C}$ con agitación constante de $100 \mathrm{rpm}$ (Ayesteran, 2006).

\section{Obtención del ácido giberélico}

Después de la incubación, se filtró una alícuota de $50 \mathrm{~mL}$ a través de una membrana con poro de $0.45 \mu \mathrm{m}$ de diámetro; la porción líquida fue procesada para extraer el ácido giberélico. El filtrado fue ajustado a pH 2 con $\mathrm{HCl} 1 \mathrm{M}$, colocado en un embudo de separación y se agregaron 20 $\mathrm{mL}$ de acetato de etilo concentrado y posteriormente agitarlo vigorosamente durante 5 minutos, dejándolo reposar y lograr la separación de las fases. La fase orgánica se hizo pasar a través de sulfato de sodio anhidro para almacenarla posteriormente en un matraz de bola; la fase acuosa restante fue colocada en el embudo de separación para nuevamente adicionarle $20 \mathrm{~mL}$ de acetato de etilo concentrado; este proceso se realizó 5 veces. La fase orgánica contenida en el matraz de bola fue llevada al rotavapor, para evaporar el disolvente a $35^{\circ} \mathrm{C}$ y obtener el sólido deseado.

\section{Caracterización}

Al producto obtenido de la fermentación durante 7 días se le realizó un análisis por cromatografía en placa, la cual fue comparada con el producto comercial y analizada en el espectrofotómetro de UV visible (SHIMADZU, UV-160A) para determinar su longitud de onda y complementar la caracterización con un análisis en el espectrofotómetro de Infrarrojo (Nicolet, AVATAR 360 FT-IR).

\section{Análisis del ácido giberélico estándar y comercial}

Al ácido giberélico estándar (Riedel-de Haen, Lot 3251X) se le realizó el análisis en el espectrofotómetro de Infrarrojo, utilizando $1 \mathrm{mg}$ del estándar y $50 \mathrm{mg}$ de $\mathrm{KBr}$. la pastilla fue 
preparada para ser analizada en el IR. También fue realizada la curva de calibración del $\mathrm{AG}_{3}$ estándar y leída en el espectrofotómetro de UV visible para determinar la longitud de onda máxima empleando celdas de cuarzo de $1 \mathrm{~cm}$ de diámetro e indicando en el equipo la lectura de 200 a $900 \mathrm{~nm}$. Las diluciones realizadas fueron en ppm (500, $300,200,100,50$ y 10) y usando como disolvente acetato de etilo.

El ácido giberélico comercial (Biogib) empleado presentó una pureza del $10 \%$, por lo que para su purificación se pesaron y disolvieron $1.0274 \mathrm{~g}$ de Biogib en $100 \mathrm{~mL}$ de agua desionizada. Ajustándolos a un pH 2 con una solución $1 \mathrm{M}$ de $\mathrm{HCl}$. Una alícuota de $50 \mathrm{~mL}$ de la solución fue colocada en un embudo de separación para adicionarle 50 $\mathrm{mL}$ de acetato de etilo y agitar inmediatamente con la finalidad de separar las fases, la fase orgánica contiene al ácido giberélico. La fase orgánica se hizo pasar a través de los cristales de sulfato de sodio anhidro, y posteriormente fue evaporado el acetato de etilo en un rotavapor (Brinkmann, Büchi 461). Posteriormente se determinó el punto de fusión (Fisher-Johns) y se realizó una cromatografía en placa fina empleando como sistema eluyente acetato de etilo-acetona-diclorometano (9:1:1) y finalmente la placa fue observada con la lámpara de luz UV. Se realizó el análisis en el espectrofotómetro de Infrarrojo, pesándose $0.0010 \mathrm{~g} \mathrm{del} \mathrm{AG}_{3}$ comercial y 0.0529 $\mathrm{g}$ de $\mathrm{KBr}$, y determinó la longitud de onda máxima empleando el espectrofotómetro de UV visible (8)

\section{Obtención del lodo residual y tratamiento}

El lodo residual municipal (LRM) se obtuvo de una Planta Tratadora de Aguas Residuales en Toluca, Estado de México, donde se tomó una muestra de $6 \mathrm{~L}$ de lodo antes de la adición del polímero y de la entrada al filtro prensa. Se esterilizó en una autoclave a presión (UL, modelo No. $1925 \mathrm{~K}$ ) a $15 \mathrm{lb}$ durante 25 minutos y posteriormente se realizó la prueba de esterilidad, para la cual, se prepararon cajas petri con agar nutritivo y se sembró la muestra de lodo por estrías y se incubaron las cajas inoculadas durante 24 horas a $37^{\circ} \mathrm{C}$.

\section{Caracterización del lodo residual}

Se determinó el pH con un potenciómetro (CONDUCTRONIC pH 120) (15), el porcentaje de materia orgánica empleando el método de Walkey y Black (15) el contenido de nitrógeno total por el método de Kjeldahl y el contenido de humedad relativa (15). La concentración de metales pesados (Cr, Zn, Ni, Pb, Cu, Cd) se determinó empleando la técnica de absorción atómica utilizando el equipo de ab- sorción atómica (Perkin Elmer, Analist 200, estos metales se midieron a una $\lambda=357.87,213.86,232,283.3,324.75$, 228.80 respectivamente).

\section{Cultivo del hongo y producción de $\mathrm{AG}_{3}$ empleando LRM y MCE}

Ambos sustratos se usaron al $100 \%$, variando el tiempo de incubación, se emplearon matraces con $100 \mathrm{~mL}$ del sustrato tanto del lodo residual como del medio de cultivo estándar, se esterilizaron y posteriormente se inoculó cada matraz con el contenido de un tubo de cultivo (colonias de Gibberella fujikuroi) y se incubaron durante 3, 8,13 y 30 días a $28^{\circ} \mathrm{C}$ en fermentación sumergida y agitación constante. Después del tiempo de incubación correspondiente se realizó la extracción del producto formado en cada muestra realizando cinco lavados con acetato de etilo, se evaporó el disolvente en el rotavapor y finalmente el sólido fue disuelto en metanol y llevado a un volumen final de $10 \mathrm{~mL}$.

\section{Determinación cuantitativa del $\mathbf{A G}_{3}$ producido en LRM y MCE}

Para la determinación cuantitativa de ácido giberélico producido se empleó cromatografía líquida de alta resolución HPLC (Varian, 9050,9012), para la separación del produc-

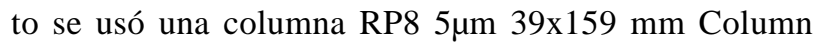
W2197 H 005, empleando como fase móvil $35 \%$ metanol, $65 \%$ agua $\mathrm{pH} 4$ (ácido fosfórico $1 \%$ ), con un flujo de $1 \mathrm{~mL} / \mathrm{min}$, ciclo de $10 \mu \mathrm{L}$, volumen de inyección $50 \mu \mathrm{L}$, detector UV a $256 \mathrm{~nm}$, intervalo de concentraciones 100 mg/L - 1000 mg/L. La cuantificación del ácido se realizó mediante comparación con una curva de calibración obtenida para un patrón de ácido giberélico de Riedel-de Haen, Lot $3251 \mathrm{X}$.

Para la curva de calibración se prepararon soluciones de 500, 300, 200, 100, 50 y 10 ppm diluidas en metanol.

\section{Resultados y discusión}

\section{Características de la cepa de Gibberella fujikuroi}

La cepa de Gibberella fujikuroi utilizada se muestra en la figura 1A, la cual, creció formando colonias sobre el medio de cultivo PDA. Las colonias en la etapa inmadura presentaron un micelio de color blanco y aspecto algodonoso, y con la madurez del cultivo, las colonias presentaron un color anaranjado o violeta en la base del crecimiento. 
En la figura 1B, se observan las características microscópicas del hongo al aplicar azul-algodón-lactofenol, también se observan estructuras miceliares alargadas con ramificaciones septadas. No se observaron esporas, lo que coincide con la información bibliográfica (8), que menciona al hongo como un tipo de cepa no formadora de esporas.

\section{Caracterización del producto obtenido del MCE}

La figura $1 \mathrm{C}$, muestra una cromatografía en placa del producto obtenido en MCE durante siete días de fermentación sumergida (a), en donde la altura del producto fue de $3.6 \mathrm{~cm}$ y la altura alcanzada por el disolvente fue de 6.5 cm, obteniendo una $\mathrm{R}_{\mathrm{F}}=0.55$. La $\mathrm{R}_{\mathrm{F}}$ del producto comercial (b) dio el mismo valor, ya que la distancia recorrida por el producto fue igual a la alcanzada por la muestra obtenida en MCE. Ambas cromatografías presentaron una $\mathrm{R}_{\mathrm{F}}$ similar a la obtenida para el estándar (0.52).

\section{Caracterización del $\mathrm{AG}_{3}$ estándar y comercial}

La figura 1D muestra la cromatografía en placa realizada al estándar de ácido giberélico, donde la altura obtenida por la muestra fue de $3.5 \mathrm{~cm}$ y la del sistema eluyente igual a $6.7 \mathrm{~cm}$, resultando una $\mathrm{R}_{\mathrm{F}}=0.52$, dato aplicado como punto de referencia para la identificación del $\mathrm{AG}_{3}$ presente en el producto obtenido en el MCE con 7 días de incubación.
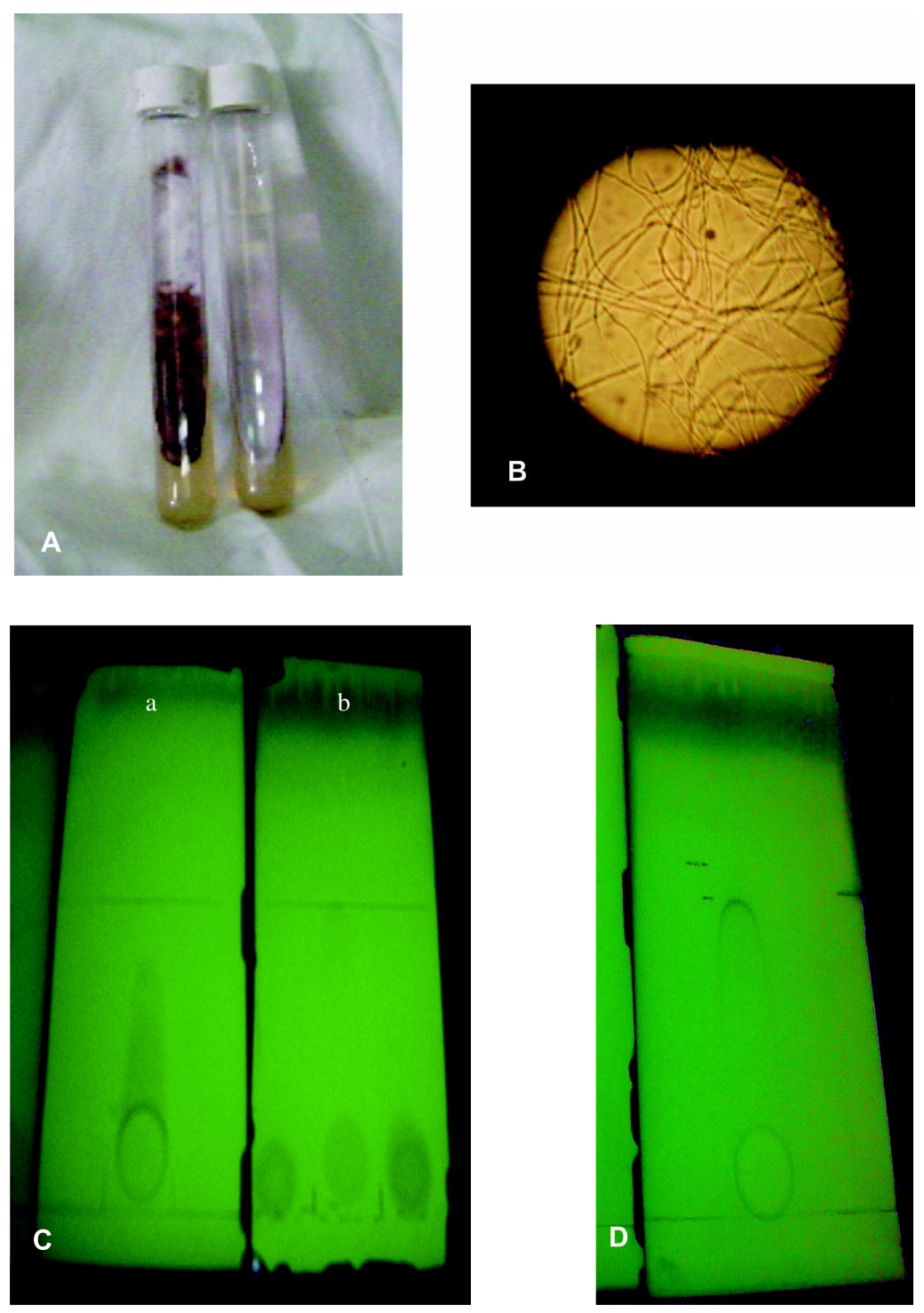

Figura 1. A: Aspecto de la Gibberella fujikuroi durante su desarrollo. B: Características microscópicas de la Gibberella fujikuroi. C: Cromatografía del producto extraído (a) y el ácido giberélico comercial (b). D: Cromatografía del ácido giberélico estándar. 


\section{Espectro IR del $\mathrm{AG}_{3}$ extraído de MCE y LRM}

La figura $2 \mathrm{~A}$ y $2 \mathrm{~B}$, muestra el espectro en el IR del $\mathrm{AG}_{3}$ extraído del MCE y LRM respectivamente, observando que tienen semejanza con el espectro del $\mathrm{AG}_{3}$ estándar (Figura 3A) comercial (Figura 3B), pues en ellos se observa la existencia de la banda que corresponde al grupo $\mathrm{O}-\mathrm{H}$ de los ácidos carboxílicos (3500-2500 $\left.\mathrm{cm}^{-1}\right)$, y frecuentemente centrada alrededor de $3000 \mathrm{~cm}^{-1}$, así como la existencia del enlace $\mathrm{C}=\mathrm{O}\left(1720-1700 \mathrm{~cm}^{-1}\right)$ para el ácido. En relación al grupo éster, la banda muestra señales entre 1750$1700 \mathrm{~cm}^{-1}$ que corresponde al enlace $\mathrm{C}=\mathrm{O}$ de dicho grupo, además se identifican señales correspondientes al enlace C-H de los alcanos (2800 y $3000 \mathrm{~cm}^{-1}$ ) y una señal en 2400 $\mathrm{cm}^{-1}$ que indica la presencia de $\mathrm{CO}_{2}$. La cantidad de sustancia química en la muestra contribuye a los anchos de los picos, y hace que el enlace de hidrógeno se presente como bandas anchas, las cuales están asociadas con el grupo O-H lo cual se observa en la figura $2 \mathrm{~A}$. Cuando la muestra es muy diluida el enlace O-H presenta bandas nítidas y más débiles tal como se muestra en la figura 2B Smith (16); estos resultados cualitativos indican que la cepa de Gibberella fujikuroi es productora de ácido giberélico en el medio de cultivo estándar y en el lodo residual municipal.

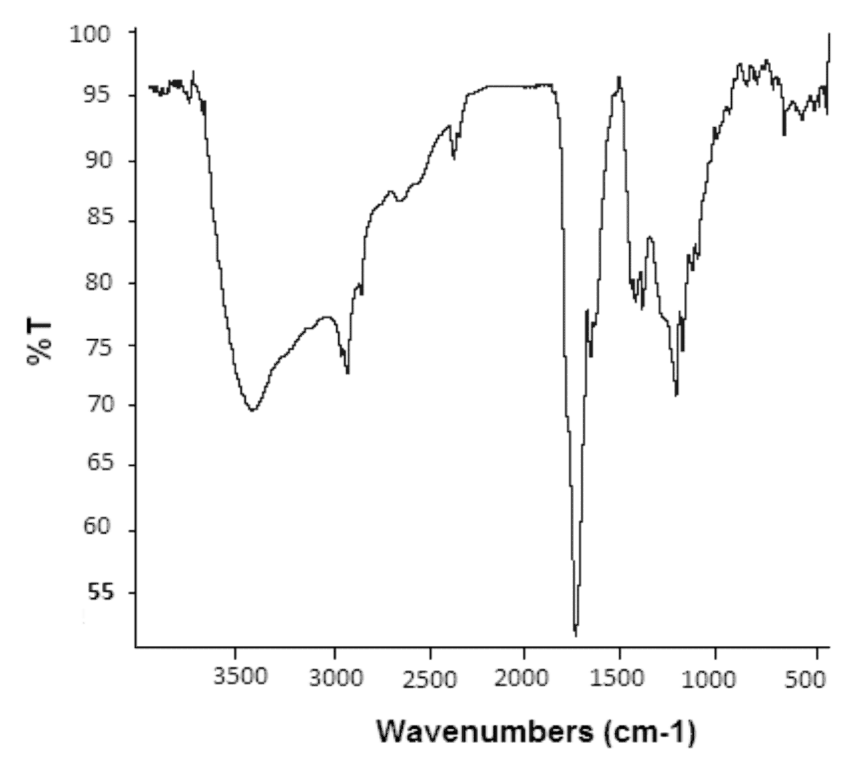

A

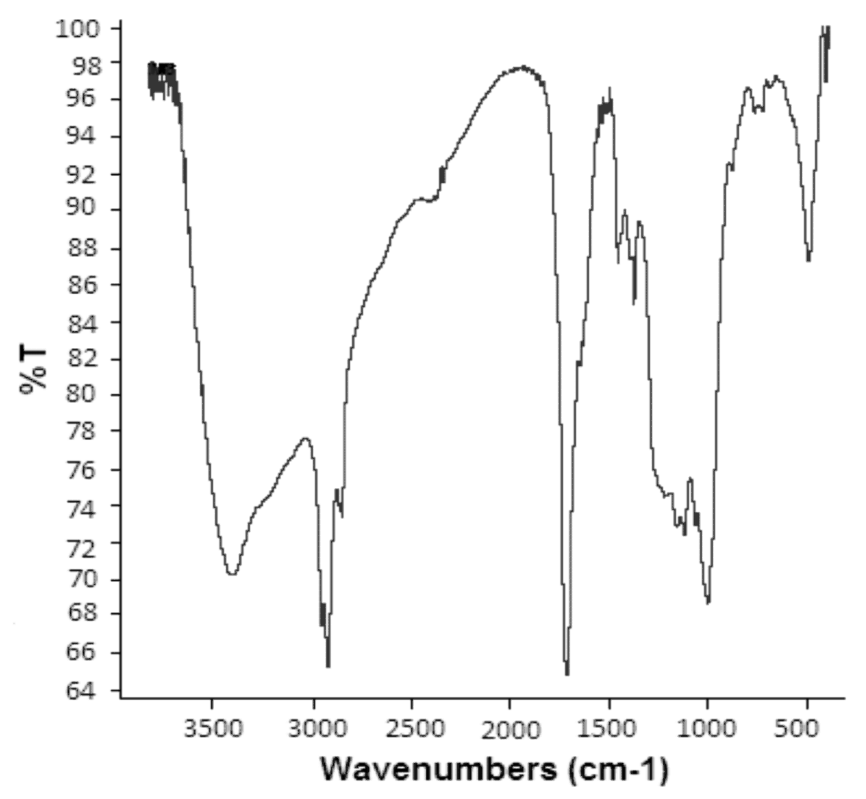

B

Figura 2. A: Espectro en el IR de $\mathrm{AG}_{3}$ extraído del MCE. B: Espectro en el IR de $\mathrm{AG}_{3}$ extraído del LRM. 
En la figura 3A y 3B se muestran los espectros del ácido giberélico estándar y comercial obtenidos en el espectrofotómetro de Infrarrojo. En ambos espectros se pueden observar las bandas que corresponden al grupo $\mathrm{O}-\mathrm{H}$ de los ácidos carboxílicos $\left(3500-2500 \mathrm{~cm}^{-1}\right)$, así como la existencia del enlace $\mathrm{C}=\mathrm{O}\left(1720-1700 \mathrm{~cm}^{-1}\right)$ para el ácido. En relación al grupo éster, la banda muestra seña- les entre $1750-1700 \mathrm{~cm}^{-1}$ que corresponde al enlace $\mathrm{C}=\mathrm{O}$ de dicho grupo, en ambos espectros se identifican señales correspondientes al enlace C-H de alcanos (entre 2800 y $\left.3000 \mathrm{~cm}^{-1}\right)$. El espectro del $\mathrm{AG}_{3}$ comercial presenta más intensa la banda correspondiente al grupo $\mathrm{CO}_{2}\left(2400 \mathrm{~cm}^{-}\right.$ 1) Smith (16).
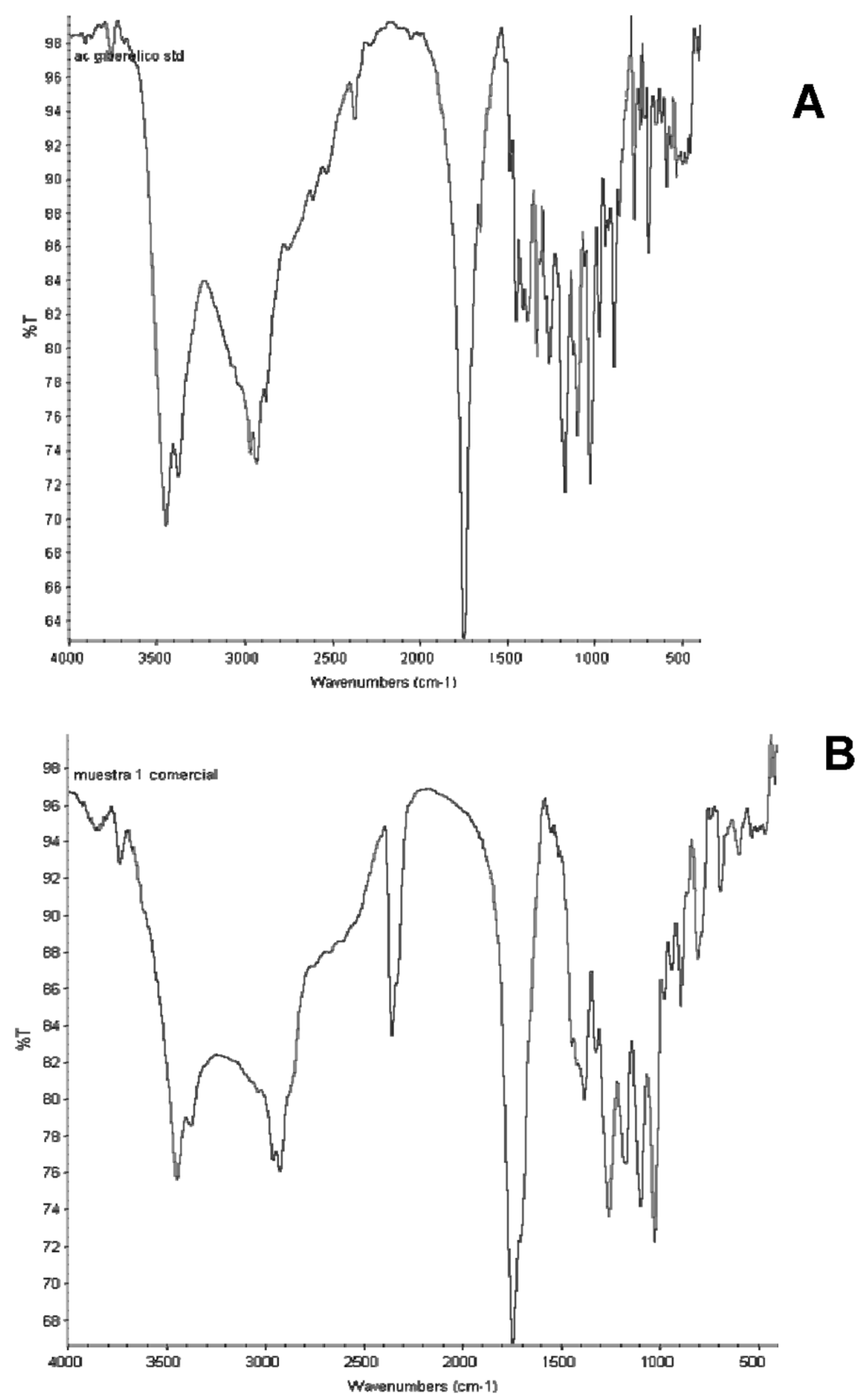

Figura 3. A: IR del ácido giberélico estándar. B: IR del ácido giberélico comercial. 
La figura 4A y 4B exponen la longitud de onda determinada en el UV visible para el estándar y producto comercial de ácido giberélico respectivamente. La longitud de onda fue de $256 \mathrm{~nm}$, ambos presentan una intensidad de radiación similar, siendo de 0.25 para el estándar y 0.2 para el ácido giberélico comercial. Los resultados confirmaron que el producto comercial podía ser usado como control positivo.

\section{Tratamiento y caracterización del lodo residual}

En relación al tratamiento realizado al lodo residual municipal la prueba de esterilidad presentó nulo crecimiento de microorganismos en el agar nutritivo, confirmando que es seguro el proceso de esterilización realizado a 15 lb durante 25 minutos.

En la caracterización del lodo residual, éste se ajustó a pH ácido, condición óptima para el crecimiento del hongo. Las características fisicoquímicas determinadas al lodo residual con ajuste ácido y alcalino se muestran en el tabla 1.

En el LRM alcalino y en el LRM ácido, con relación al porcentaje de materia orgánica fue similar y de acuerdo al Instituto Nacional de Investigaciones Pecuarias (INIA), (Moreno-Dahme, 1970) citado en Valdés y Medina (15) el sustrato se clasifica como extremadamente rico en materia orgánica $(5.20 \%$ m/v). Así mismo, el nitrógeno en las mues-

Estándar de ácido giberélico

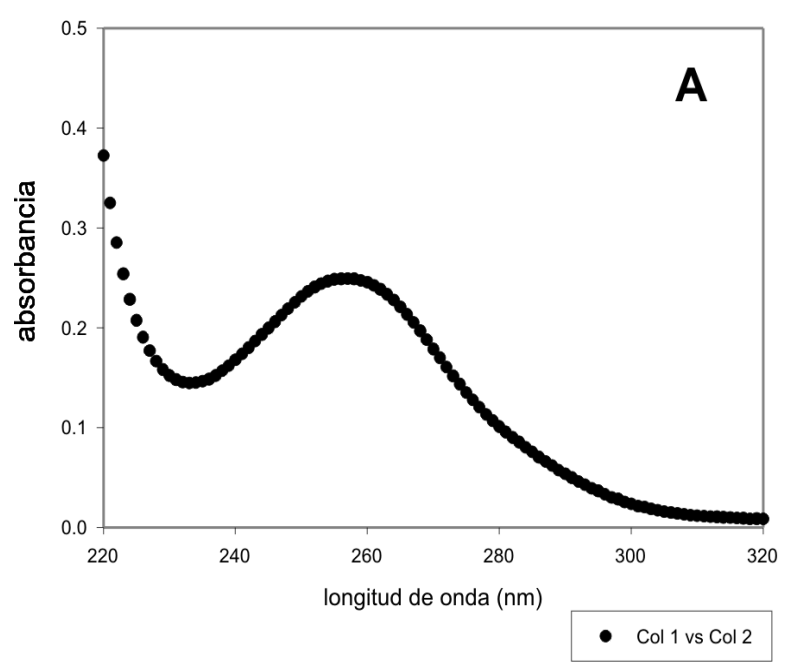

Tabla 1. Parámetros físico-químicos del lodo residual municipal

\begin{tabular}{lcc}
\hline Parámetro & $\begin{array}{c}\text { LRM } \\
\text { ácido }\end{array}$ & $\begin{array}{c}\text { LRM } \\
\text { alcalino }\end{array}$ \\
\hline $\mathrm{pH}$ & 4.0 & 8.2 \\
Materia orgánica $(\% \mathrm{~m} / \mathrm{v})$ & 5.2 & 5.3 \\
Nitrógeno total $(\% \mathrm{~m} / \mathrm{v})$ & 0.25 & 0.24 \\
Humedad relativa $(\% \mathrm{~m} / \mathrm{v})$ & 95.6 & 95.3 \\
\hline
\end{tabular}

tras de lodo presentó concentraciones similares, siendo de $0.24 \%(\mathrm{~m} / \mathrm{v})$ y $0.25 \%(\mathrm{~m} / \mathrm{v})$ respectivamente. Estos resultados coinciden con Muhammad et al. (2008), los cuales mencionan que los lodos residuales contienen nutrientes para las plantas y MO que puede ser usada como suplemento, además se ha mostrado que la aplicación de lodos residuales mejora las propiedades físicas, químicas y biológicas del suelo.

En la tabla 2, se presenta la determinación de algunos metales pesados y la comparación con los límites máximos permisibles de estos contaminantes de acuerdo a la NOM-004-SEMARNAT-2002. Se observa que los valores obtenidos para el cromo $(588.40 \mathrm{mg} / \mathrm{Kg})$, zinc $(496.58$ $\mathrm{mg} / \mathrm{Kg})$ y cobre $(405.27 \mathrm{mg} / \mathrm{Kg})$ no rebasan los límites máximos permisibles citados en la norma oficial mexicana, la concentración de plomo $(402.88 \mathrm{mg} / \mathrm{Kg})$ rebasa el

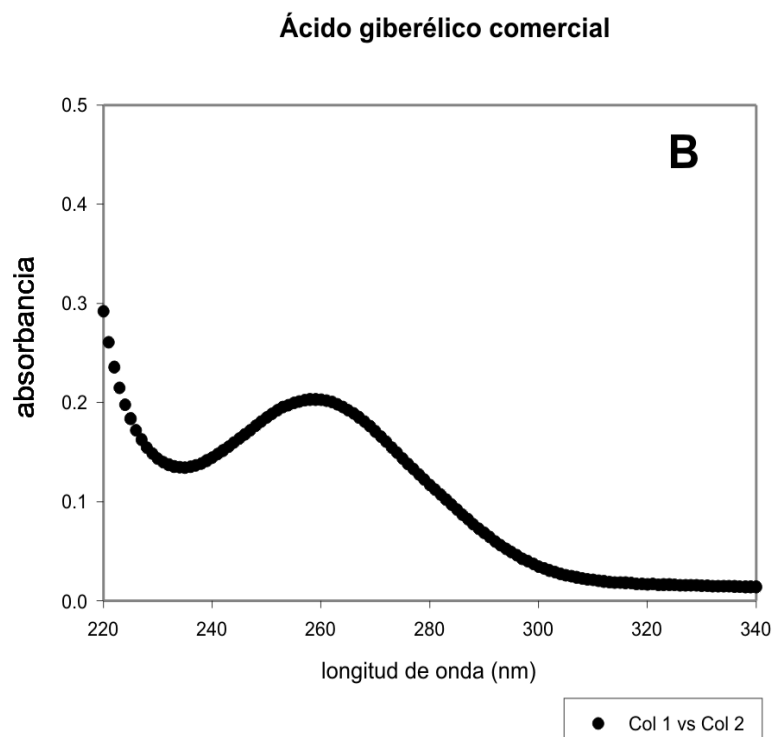

Figura 4. A: Longitud de onda para el estándar de ácido giberélico. B: Longitud de onda para el producto comercial de ácido giberélico. 
Tabla 2. Metales pesados en lodos y límites máximos permisibles.

\begin{tabular}{lcccc}
\hline & & \multicolumn{2}{c}{ NOM-004-SEMARNAT-2002 } \\
\hline Metal & $\begin{array}{c}\text { LRM alcalino } \\
\mathrm{mg} / \text { Kg en base seca }\end{array}$ & $\begin{array}{c}\text { LRM ácido } \\
\mathrm{mg} / \mathrm{Kg} \text { en base seca }\end{array}$ & $\begin{array}{c}\text { Excelentes } \\
\mathrm{mg} / \mathrm{Kg} \text { en base seca }\end{array}$ & $\begin{array}{c}\text { Buenos } \\
\mathrm{mg} / \mathrm{Kg} \text { en base seca }\end{array}$ \\
\hline Cromo & 547.01 & 588.40 & 1200 & 3000 \\
Zinc & 576.96 & 496.58 & 2800 & 7500 \\
Níquel & 590.93 & 687.71 & 420 & 420 \\
Plomo & 363.34 & 402.88 & 300 & 840 \\
Cobre & 404.75 & 405.27 & 1500 & 4300 \\
Cadmio & 111.79 & 159.28 & 39 & 85 \\
\hline
\end{tabular}

límite de $300 \mathrm{mg} / \mathrm{Kg}$, aún así, está dentro del rango considerado como bueno para usarse $(840 \mathrm{mg} / \mathrm{Kg})$. En este trabajo, se encontró que níquel y cadmio tuvieron concentraciones de $687.71 \mathrm{mg} / \mathrm{Kg}$ y $159 \mathrm{mg} / \mathrm{Kg}$ respectivamente, y rebasaron los límites permisibles; sin embargo, los valores altos de estos metales no afectaron el crecimiento del hongo y por lo tanto, la producción de ácido giberélico.

\section{Determinación cuantitativa de $\mathrm{AG}_{3}$ producido en LRM y MCE}

Se obtuvo un cromatograma correspondiente al ácido giberélico estándar, como se aprecia en la figura 5, el cual presenta cuatro picos durante los primeros dos minutos. El más evidente se observa a 1.091 minutos, con una concentración de $300 \mathrm{mg} / \mathrm{L}$, considerado entonces como el tiempo de retención $\left(\mathrm{t}_{\mathrm{r}}\right.$ ) para el estándar a $256 \mathrm{~nm}$. Los picos detectados después del tiempo de retención son debido a ciertas impurezas (del mismo producto, el cual, tiene una pureza del $99 \%$ ) presentes en la solución. Estos datos se tomaron como referencia para revelar la presencia de este compuesto en las otras muestras.

Las muestras provenientes del LRM y MCE fueron analizadas y contrastadas con el tiempo de retención del estándar (1.091 minutos). En el cromatograma correspondiente al producto obtenido del LRM (Figura 6), se observa un pico a 1.083 minutos y en el cromatograma para el producto obtenido del MCE (Figura 7) el pico se muestra a 1.075 minutos, estas señales corresponden a 8 días de fermentación con tiempos cercanos al tiempo de retención determinado para el estándar; por lo tanto, la cepa empleada en este trabajo produjo $\mathrm{AG}_{3}$ en ambos sustratos. Los picos que ambos cromatogramas presentan antes y después del tiempo de retención pueden corresponder a diferentes com- puestos presentes en el lodo u otros metabolitos producidos por el hongo.

Para la producción de $\mathrm{AG}_{3}$ en LRM y MCE en función del tiempo de incubación en fermentación sumergida, las tablas 3 y 4 exhiben los resultados obtenidos en cada uno de los sustratos.

Comparando ambas tablas puede observarse que la concentración fue mayor en el LRM a los 3 días y 8 días, con una concentración de $57.61 \mathrm{mg} / \mathrm{L}$ y $258.59 \mathrm{mg} / \mathrm{L}$ respecti-

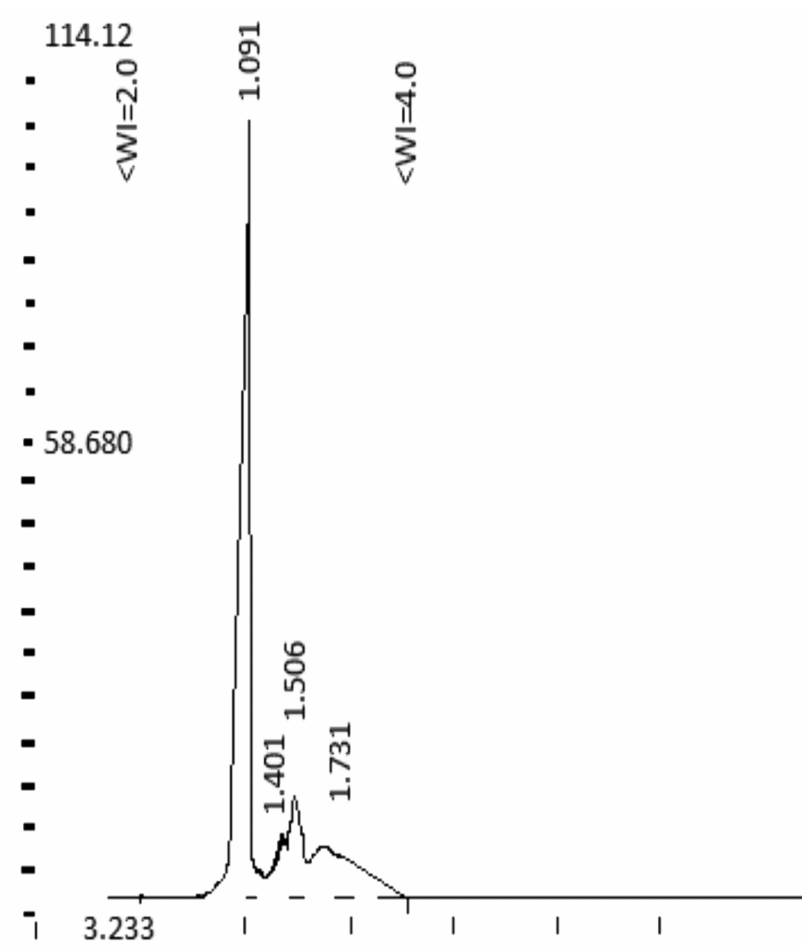

Figura 5. Cromatograma y tiempo de retención para el ácido giberélico estándar. 


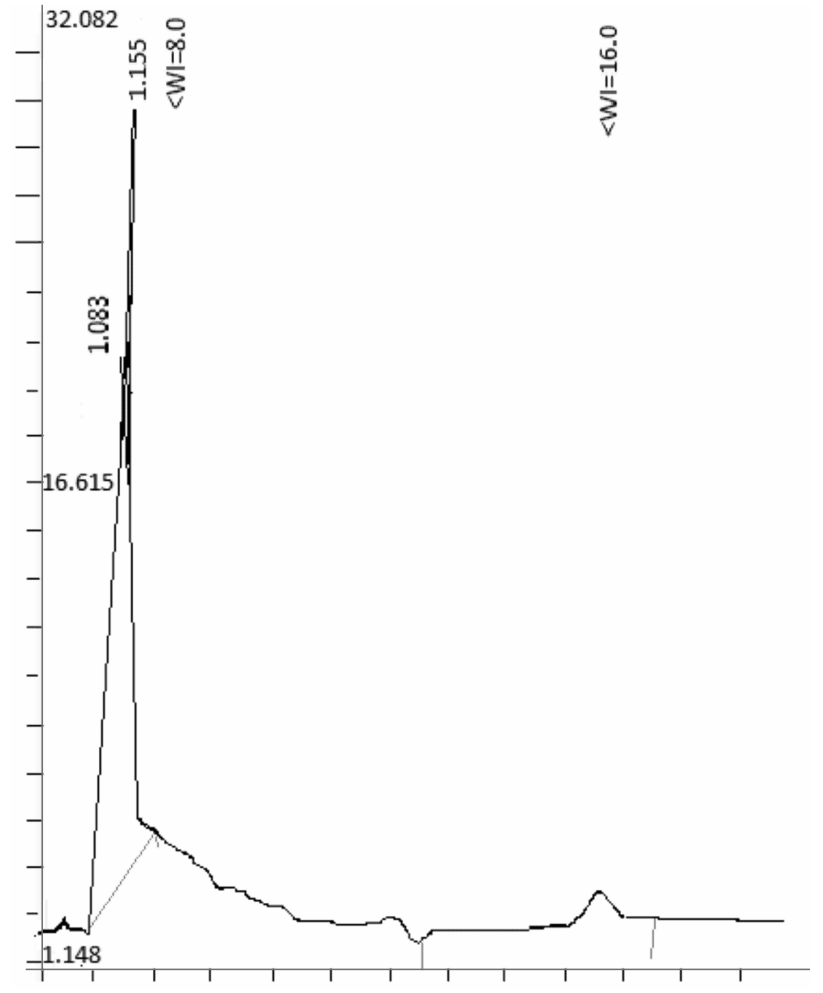

Figura 6. Cromatograma para el producto obtenido del LRM.

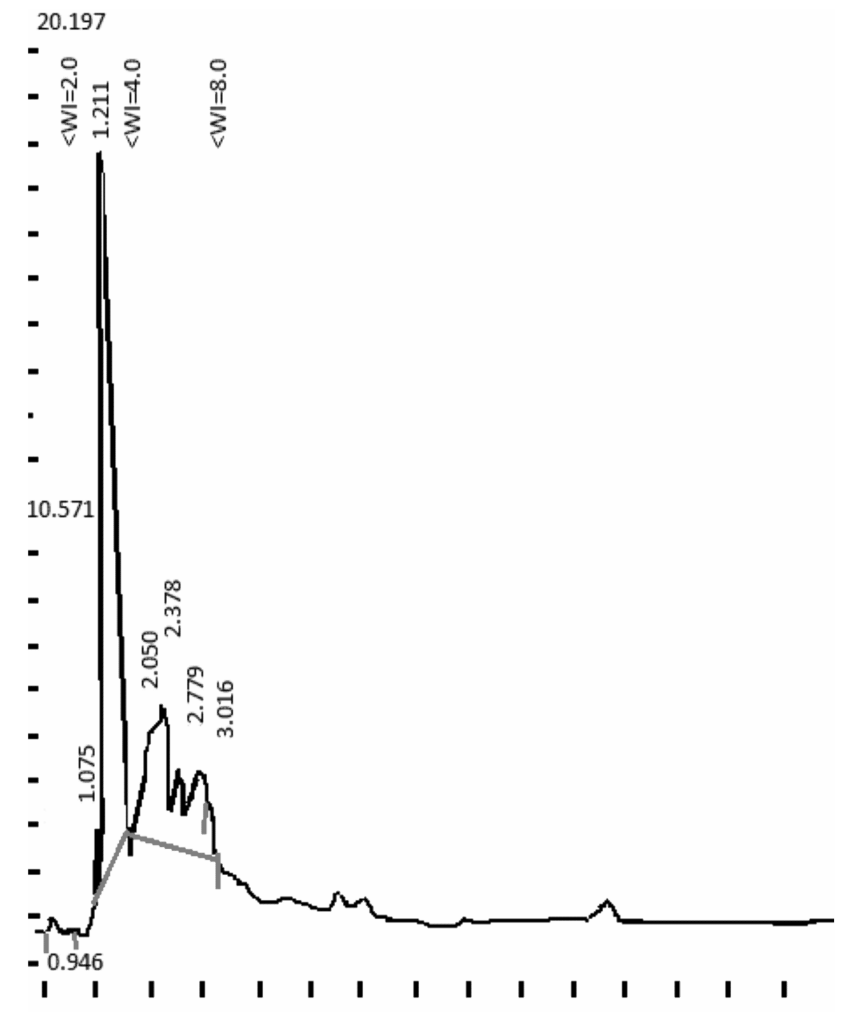

Figura 7. Cromatograma para el producto obtenido del MCE. vamente, y a los 13 días y 30 días aumenta lentamente en comparación con los rendimientos obtenidos en el MCE. Obteniéndose una concentración máxima de $\mathrm{AG}_{3}$ igual a $460.06 \mathrm{mg} / \mathrm{L}$ a los 30 días de fermentación sumergida. Ayesteran (2006) reportó una máxima concentración de ácido giberélico igual a $38 \mathrm{mg} / \mathrm{L}$ en 4 días de cultivo en lodo residual, rendimiento menor al obtenido en este trabajo a los 3 días de incubación en el lodo residual municipal.

\section{Tabla 3. Concentración de $\mathrm{AG}_{3}$ en $\mathrm{LRM}$ a diferentes} días de incubación.

\begin{tabular}{cc}
\hline \multicolumn{2}{c}{ LRM } \\
\hline Días & Concentración promedio $(\mathrm{mg} / \mathrm{L})$ \\
\hline 3 & 57.61 \\
8 & 258.59 \\
13 & 305.98 \\
30 & 460.06 \\
\hline
\end{tabular}

Tabla 4. Concentración de $\mathrm{AG}_{3}$ en el MCE a diferentes días de incubación.

\begin{tabular}{cc}
\hline \multicolumn{2}{c}{ MCE } \\
\hline Días & Concentración promedio $(\mathrm{mg} / \mathrm{L})$ \\
\hline 3 & 45.16 \\
8 & 220.82 \\
13 & 425.87 \\
30 & 1014.46 \\
\hline
\end{tabular}

La concentración de $\mathrm{AG}_{3}$ obtenida en el LRM se debe al contenido de MO (Tabla 1) metabolizada por el hongo ya que con base en Valdés y Medina (2005), es un sustrato extremadamente rico en este nutriente, así mismo, el pH y la cantidad de nitrógeno presente en el lodo favoreció estos resultados; Ayesteran (2006) sugiere que para un mejor rendimiento en la producción de ácido giberélico empleando lodo residual, el $\mathrm{pH}$ del lodo debe ser ácido, igual que el medio de cultivo estándar ( $\mathrm{pH} 4.0$ ), condición que se realizó desde el inicio de este trabajo. En relación al nitrógeno, Ayesteran (2006) reporta $240 \mathrm{mg} / \mathrm{L}$ de lodo, mientras que el LRM empleado en el presente trabajo presentó un valor de $2500 \mathrm{mg} / \mathrm{L}$, concentración diez veces mayor. Por lo tanto, a mayor concentración de nitrógeno, mayor crecimiento del hongo y cuando la fuente de nitrógeno se agota, el crecimiento exponencial del hongo decae y la producción de metabolitos secundarios se incrementa. Sin embargo, Gelmi et al. (2000) mencionan que con altas concentraciones, la fase de crecimiento se prolonga y la 
producción de la hormona se puede retardar, éste comportamiento es observado en los rendimientos de $\mathrm{AG}_{3}$ obtenidos (Tabla 3) con los reportados para el MCE (Tabla 4). La diferencia en cuanto a los rendimientos, teniendo en cuenta que se empleó un medio nutritivo totalmente diferente al MCE, esto es debido principalmente a que existe una descomposición del ácido giberélico que promueve la formación de ácido giberelénico, así como productos intermedios como ácido iso-giberélico. Descomposición que se da en medios acuosos, ligeramente ácidos; considerando la posibilidad que parte del ácido producido en la fermentación sumergida se degradará en otras sustancias antes de ser analizado (Pierotty et al. 2006).

\section{Conclusiones}

El lodo residual municipal obtenido de una planta tratadora de aguas residuales puede emplearse para producir ácido giberélico al ajustar el valor del $\mathrm{pH}$, ya que la concentración de materia orgánica y nitrógeno son suficientes para el cultivo del hongo Gibberella fujikuroi.

La mayor producción de ácido giberélico se genera en el LRM a los treinta días de fermentación sumergida, obteniéndose una concentración máxima de 460.06 mg/L.

Es posible incrementar la producción de ácido giberélico utilizando como sustrato lodo residual municipal a 460.06 $\mathrm{mg} / \mathrm{L}$ en una fermentación sumergida de 30 días, en relación a la concentración máxima obtenida por Ayesteran (2006) que fue en tres días de $38 \mathrm{mg} / \mathrm{L}$.

El criterio empleado en la concentración del inoculo es confiable, ya que al comparar la concentración de $\mathrm{AG}_{3}$ producido en ambos sustratos $(\mathrm{LRM}=258.59 \mathrm{mg} / \mathrm{L}$ y MCE $=220.82 \mathrm{mg} / \mathrm{L}$ ) y los tiempos de retención (LRM = 1.083 minutos y $\mathrm{MCE}=1.075$ minutos) a los 8 días de fermentación se obtienen datos similares.

\section{Financiación}

Con apoyo financiero de la Universidad Autónoma del Estado de México bajo los proyectos con clave: 2825/ 2009U y 2971/2010SF.

\section{Conflicto de intereses}

Los autores no presentan conflicto de intereses

\section{Referencias}

1. Machado MC, Oishi BO, Ashok P, Soccol CR. Kinetics of Gibberella fujikuroi Growth and Gibberellic Acid
Production by Solid-State Fermentation in a PackedBed Column Bioreactor. Biotechnology Progress 2004; 20, 1449-1453

2. Kawaide H. Biochemical and Molecular Analyses de Gibbeerellin in Fungi. Bioscience, Biotechnology and Biochemistry 2006; 70 (3): 583-590

3. Pierotty CD, Otálvaro AA, Algecira EN. Producción de acido giberélico utilizando biocatalizadores soportados en matrices porosas. Universitas Scientiarum 2006; 11 (2): 41-50

4. Sanfeliu M, Teófilo JV, Manuel M, Boix A. 2005. Contaminación y medio ambiente: Santiago (Chile), Castellón (Epaña) 1998-2002. pp. 507

5. Gelmi C, Pérez CR, González M, Agosin E. Solid substrate cultivation of Gibberella fujikuroi on an inert support. Process Biochemistry 2000; 35: 1227-1233

6. Durán PE, Molina J, Marco A, Brito A, Robles MF. Gibberellic Acid Production by Free and Immobilized Cells in Different Culture Systems. Applied Biochemistry and Biotechnology 2004; 113-116: 381-388

7. Nhujak T, Srisa AM, Kalampakorn K, Tolieng V, Petsom A. Determination of Gibberellic Acid in Fermentation Broth and Commercial Products by Micellar Electrokinetic Chromatography. Journal of Agricultural and Food Chemistry 2005; 53, 1884-1889

8. Ayesteran L.M. Lodos residuales para la obtención de ácido giberélico por Gibberella fujikoroi para la aplicación en la agricultura. Trabajo de Grado de Maestría. Facultad de Química. UAEM. Toluca, México, 2006, 80 p.

9. Colín CA, López CR, Olea CO, Barrera DC, Valdez FC. Products generated from the termal treatment of sewage sludge. IWA Specialised conference on sludge management: regulation, treatment, utilisation and disposal 2001, 164-171

10. Oropeza GN. Lodos residuales: estabilización y manejo. Caos Conciencia 2006; I, 51-58

11. Rulkens W. Sewage Sludge as a Biomass Resource for the Production of Energy: Overview and Assessment of the Various Options. Energy \& Fuels 2008; 22 (1): 9-15

12. Teuber KN, Salazar SF, Alfaro VM, Valdebenito BA. Efecto de Diferente Dosis de Lodo de la Crianza de Salmones en el Cultivo de papa y su Efecto Residual 
en Ballica Anual. Agricultura Técnica 2006; 67 (4): 393-400

13. Dirkzwager AH, Hermite PL. Sewage sludge, treatment and use, New developments, technological aspects and environmental effects. Elsevier Science Publishers ltd, Crown House, Linton Road, England. 1990. 114-184

14. Colín CA. Estudio integral de lodos residuales. Trabajo de Grado de Maestría. Facultad de Química. UAEM. Toluca, México, 1994, 125 p.

15. Valdés M, Medina JN. Ecología microbiana del suelo. Compendio práctico. Primera edición. Instituto Politécnico Nacional. México. 2005; 20-34.

16. Smith B. Infrared Spectral Interpretation, A Systematic Approach. CRC Press LLC, Printed in the United States of América.1999; 67-112.
17. Jamali MK, Kazi TG, Arain MB, Afride HI, Jalbani N, kandhro GA, Shah AQ, Baig JA. Heavy metal accumulation in different varieties of wheat (Triticum aestivum L.) grown in soil amended whit domestic sewage sludge. Journal of Hazardous Materials 2008: 1-6

18. NOM-004-SEMARNAT-2002. 2002. Protección Ambiental. Lodos y Biosólidos. Especificaciones y límites máximos permisibles de contaminantes para su aprovechamiento y disposición final. Diario Oficial de la Federación. 15 de agosto del 2003

19. Tirado M. Nueva perspectiva en el reuso de lodos residuales. http://www.cepis.org.pe/bvsaidis/aresidua/ i-160.pdf. Consultado el 6 de octubre de 2010. 